\title{
Potential for severe airway obstruction from pediatric retropharyngeal abscess
}

\author{
This article was published in the following Dove Press journal: \\ International Medical Case Reports Journal \\ Number of times this article has been viewed
}

\author{
Michelle M LeRiger ${ }^{1,2}$ \\ Veronica Miler ${ }^{3}$ \\ Joseph D Tobias ${ }^{3}$ \\ Vidya T Raman ${ }^{3}$ \\ Charles A Elmaraghy ${ }^{4,5}$ \\ Kris R Jatana ${ }^{4,5}$ \\ 'Division of Pediatric Anesthesiology, \\ Omaha Children's Hospital \& \\ Medical Center, ${ }^{2}$ Department of \\ Anesthesiology, University of \\ Nebraska Medical Center, Omaha, \\ NE, ${ }^{3}$ Department of Anesthesiology \\ and Pain Medicine, ${ }^{4}$ Department \\ of Pediatric Otolaryngology- \\ Head \& Neck Surgery, Nationwide \\ Children's Hospital, ${ }^{5}$ Department \\ of Otolaryngology-Head and Neck \\ Surgery, Wexner Medical Center \\ at Ohio State University, Columbus, \\ $\mathrm{OH}, \mathrm{USA}$
}

\begin{abstract}
Retropharyngeal abscesses in the pediatric population can cause severe respiratory distress. We report a rare case of significant airway obstruction in a 14-month-old patient requiring rapid, emergent tracheotomy after attempts at endotracheal intubation by an experienced airway surgeon were unsuccessful. The patient was diagnosed with streptococcal pharyngitis 9 days prior to presentation to our facility and was being treated with amoxicillin. Prompt diagnosis, communication, and appropriate multidisciplinary airway management can lead to successful outcomes even in these severe cases.
\end{abstract}

Keywords: pharyngitis, retropharyngeal abscess, emergent tracheotomy, pediatric airway obstruction, respiratory distress

\section{Introduction}

Retropharyngeal abscesses (RPAs) are deep neck space infections that have the potential to deteriorate into life-threatening airway obstruction. The peak incidence occurs between 3 and 5 years of age, before the lymph nodes in this potential space atrophy, making abscess formation much less common with age. Abscess formation is an infrequent complication of an upper respiratory tract infection, resulting from suppuration of the retropharyngeal lymph nodes, which drain the nasopharynx and oropharynx.

The retropharyngeal space is a potential space located posterior to the pharynx, larynx, and trachea. It extends superiorly from the base of the skull and inferiorly to the bifurcation of the trachea in the mediastinum. The space is bound anteriorly by the buccopharyngeal fascia, laterally by the carotid sheaths and parapharyngeal spaces, and posteriorly by the alar fascia of the deep cervical fascia. The space is fused down the midline and contains two chains of lymph nodes extending down each side. The nodes in these deep neck spaces communicate, allowing bacteria to spread to other nodes. Once infected, the nodes may progress through three progressive stages: cellulitis, phlegmon, and abscess.

Complications of RPA can result from a mass effect and compression of adjacent structures, notability the larynx and trachea. With airway compression, it is critical to have a skilled and experienced multidisciplinary team managing these children to achieve optimal outcomes. We present a 14-month-old with a recent history of streptococcal pharyngitis who subsequently developed a large RPA causing severe acute airway compromise necessitating emergent tracheostomy. Both institutional review board approval and consent from parent/legal guardian were obtained for purposes of publication.
Correspondence: Kris R Jatana Department of Pediatric OtolaryngologyHead \& Neck Surgery, Nationwide Children's Hospital, 555 South 18th Street, Suite 2A, Columbus, $\mathrm{OH} 43205$, USA

Tel + I 6147226040

Fax + I 6I47226609

Email Kris.Jatana@nationwidechildrens. org 


\section{Case presentation}

A 14-month-old, 8-kg, routinely vaccinated male was transferred to a tertiary care pediatric medical center with concerns for respiratory distress. He had a 1-week history of fever, decreased neck mobility, and decreased oral intake. The patient was diagnosed with streptococcal pharyngitis 9 days prior, and he was being treated with a course of amoxicillin. Due to worsening symptoms, his parents brought him to the nearest hospital where a lateral neck film showed a large amount of retropharyngeal thickening and airway displacement (Figure 1). His white blood cell count was elevated at $33,000 / \mathrm{mm}^{3}$. He was transported by helicopter to our institution for further management. Physical examination revealed an alert child with a muffled voice. Vitals sign consisted of a respiratory rate of 25 breaths/minute, heart rate of 161 beats/minute, and temperature of $37.3^{\circ} \mathrm{C}$. He was maintaining oxygen saturations of $100 \%$ on room air. Oropharyngeal examination showed significant bulging of the posterior pharynx. Further imaging was delayed until the airway was secured.

The necessary airway equipment as well as surgical instruments for tracheotomy was set up prior to the patient entering the operating room. In an attempt to keep the child calm and to avoid any unnecessary manipulation of the airway, he was transported to the operating room in his mother's arms. Anesthesia was induced by slow, controlled inhalation of sevoflurane in nitrous oxide and oxygen with the goal of maintaining spontaneous ventilation. After ensuring an adequate depth of anesthesia and properly

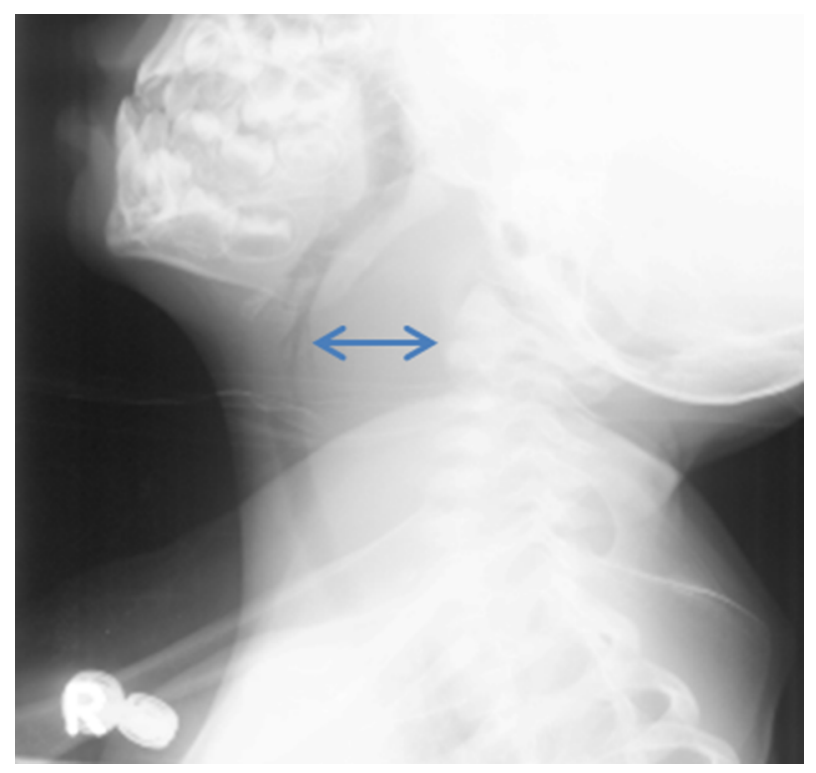

Figure I Lateral neck X-ray demonstrating significant retropharyngeal thickening (blue arrow) with anterior displacement of airway. positioning the patient, the pediatric otolaryngology team proceeded with direct laryngoscopy. The epiglottis could be visualized, but the posterior larynx was compressed anteriorly in such a way that the vocal cords could not even be seen (Figure 2A). Attempts at placing an endotracheal tube with a stylet as well as the rigid ventilating bronchoscope through the larynx were unsuccessful. Air exchange ceased, and bag-valve-mask ventilation was not possible. There was significant upper airway obstruction from mass effect alone (never a visible lumen), and this was not believed to be due to laryngospasm. The oxygen saturation began to decline rapidly, and the decision was made to proceed with emergent tracheotomy tube placement. A vertical skin incision followed
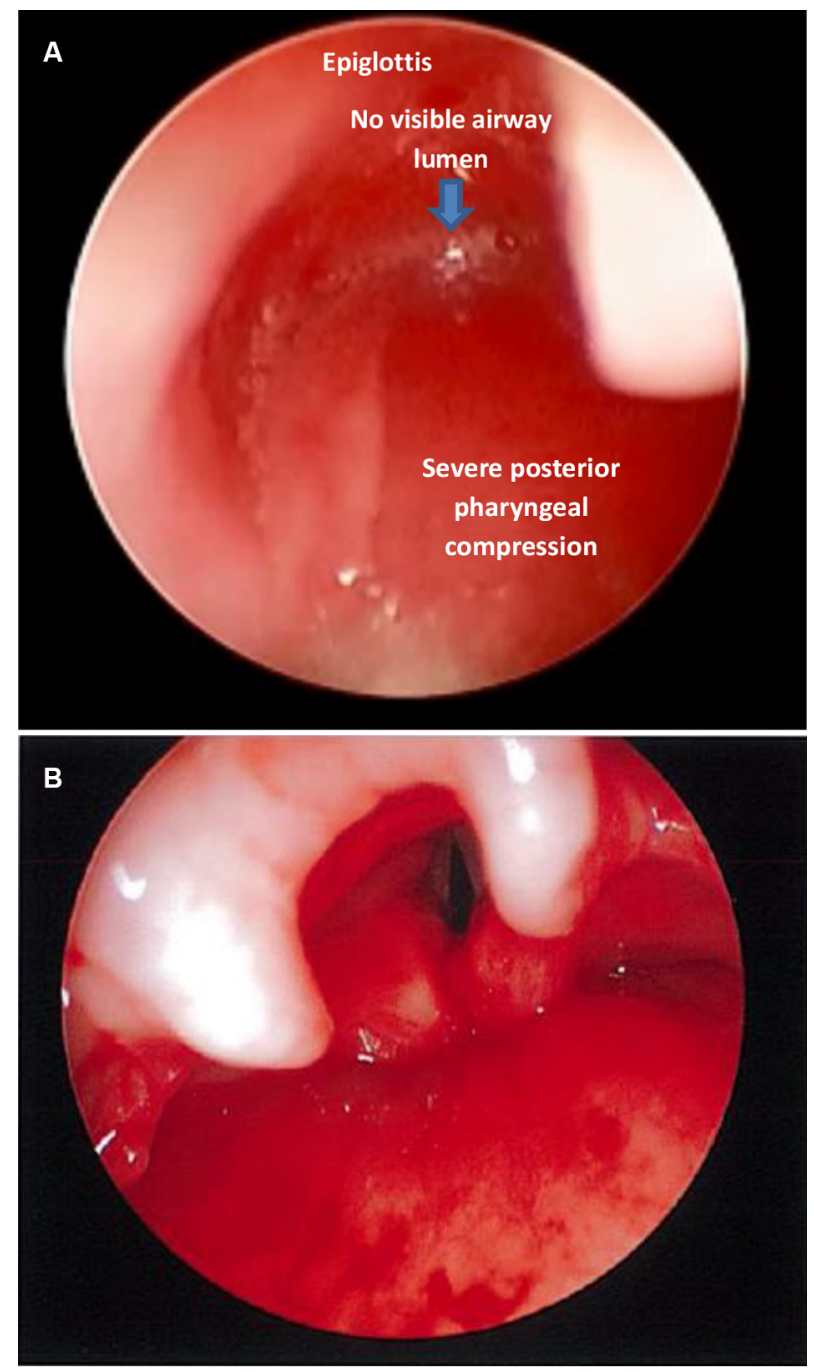

Figure 2 (A) Direct laryngoscopy showing displacement of supraglottic structures from significant retropharyngeal mass effect/compression with no visible airway lumen or visualization of vocal cords. An endotracheal tube with stylet or rigid ventilating bronchoscope could not be placed past this severe obstruction. (B) Direct laryngoscopy immediately after drainage of the large retropharyngeal abscess (airway previously secured with emergent tracheotomy), with grade I view of the larynx. 
by vertical tracheotomy was performed with a scalpel, and the airway was established within 15 seconds. Oxygen saturations quickly normalized, hemostasis was achieved, and the tracheostomy tube was secured. The posterior pharyngeal bulge was very tense, and an incision and drainage was performed with the drainage of approximately $100 \mathrm{~mL}$ of purulent fluid. Immediately following the drainage, a grade I view of the larynx was obtained (Figure $2 B$ ). The patient was transferred to the pediatric intensive care unit in stable condition. Postoperative computed tomography scan showed there was no mediastinal component that needed further intervention. Cultures of the abscess grew beta-hemolytic streptococcus group A. Serial bedside laryngoscopy was done to confirm that there was no re-accumulation of the abscess and improvement of pharyngeal edema before initiating tracheostomy decannulation process. The tracheostomy tube was removed on postoperative day 6 , and 2 weeks later the stoma was fully healed.

\section{Discussion}

The diagnosis of pediatric RPA can be difficult given a variety of nonspecific signs or symptoms including neck pain, neck stiffness, decreased oral intake, drooling, odynophagia, irritability, fever, malaise, pharyngeal swelling, and possibly external neck swelling. In a series of 73 patients, no airway symptoms were seen while awake; however, several patients had new or increased obstructive symptoms when asleep, so close monitoring is necessary. ${ }^{1}$ Signs suggesting airway compromise can include a muffled voice, stridor, respiratory distress, and obstructive sleep apnea. Dudas and Serwint stated that respiratory distress or stridor occurs less frequently than commonly believed and that most patients presenting with RPA do not necessarily have a "toxic" appearance. ${ }^{2}$ A retrospective study from Sydney Children's Hospital reported that respiratory compromise or drooling was present in $29 \%$ of children with RPA. ${ }^{3}$ In our case, given the degree of obstruction noted intraoperatively, it was surprising that our patient did not have more severe clinical symptoms. The lack of overt airway compromise may provide a false sense of security concerning the patency of the airway. In circumstances where the airway is considered critical, the practitioner should not delay definitive management for further imaging studies. It is imperative that a surgeon experienced in pediatric airway management is directly involved in the care of these children.

In current medical practices, tracheotomy and shortterm intubation (24-72 hours) are rarely required for the management of pediatric RPA. In a survey of the American Society of Pediatric Otolaryngology, a majority of surgeons reported that in their experience "a tracheotomy had never been required" for a pediatric RPA. ${ }^{4}$ Several studies have reviewed pediatric RPA, but there has been little need for tracheostomy in these series. A retrospective study from Sydney Children's Hospital of 21 children with RPA over a 12-year time span reported that 17 of 21 patients required surgical drainage. Ten patients required endotracheal intubation postoperatively for a mean duration of 4.45 days. This series reports only one patient requiring a tracheostomy, but did not specifically mention whether it was elective or emergent. ${ }^{3}$ Montreal Children's Hospital performed a retrospective study of pediatric RPA spanning from 1990 to 1999 . In this series, 17 (25\%) of 68 patients required surgical drainage. Two patients were admitted to the ICU for airway monitoring. One patient required 3 days of endotracheal intubation following the surgical procedure until the airway edema had subsided. No patients required a tracheotomy. ${ }^{5}$ Another series from Toronto included 46 retropharyngeal infections, and none required tracheotomy. ${ }^{6}$

A study from Boston Children's Hospital reviewed 73 patients with pediatric RPA from 1989 to 1998. The presenting symptoms varied, with the most common including fever, decreased oral intake, drooling, odynophagia, malaise, and torticollis. Other symptoms included neck pain, trismus, otalgia, headache, neck swelling, and change in voice quality. Four patients had worsening of snoring or frank obstructive sleep apnea, but no patient had respiratory compromise while awake. Eighteen patients had endotracheal intubation postoperatively for an average of 1-2 days, but there was no mention of any patient requiring a tracheotomy. ${ }^{1}$ In a retrospective study from New Zealand, surgical incision and drainage was carried out in 10 of the 24 (41.6\%) retropharyngeal infections. There was no mention of tracheostomy being performed in any of their patients. ${ }^{7}$

The role of medical versus surgical treatment for these infections continues to be debated. The mainstay of treatment for pediatric RPA for more than 100 years has been surgical incision and drainage. However, recent published pediatric reviews suggest that only $25-50 \%$ of patients require surgery. ${ }^{2}$ It has been proposed by some that all patients should be treated with intravenous antibiotics and that surgery be reserved for those who do not respond. ${ }^{1}$ However, it is difficult to be completely certain whether cases treated successfully with antibiotics were actually abscesses and not just cellulitis. Because the morbidity of transoral drainage is very low, it is common practice to proceed with surgical incision and drainage when a computed tomography scan suggests that a well-defined abscess is present. It is clear, however, that if 
any signs of airway obstruction are present, it is necessary to proceed with securing the airway and surgical drainage. Centers with a multidisciplinary team, consisting of an experienced pediatric anesthesiologist and surgical specialist in airway management, are critical to successful outcomes in these patients.

\section{Conclusion}

As seen from our case, complete airway obstruction can occur rapidly, necessitating emergent tracheotomy. Prompt diagnosis and early treatment is imperative to prevent such complications. Involvement of experienced pediatric anesthesiologists and surgeons trained in pediatric airway management, including emergent tracheotomy, is critical for optimal outcomes in severe cases. Because RPA can present with similar signs and symptoms as other upper airway infections, pediatric clinicians must always keep it in their differential diagnosis. Even in patients with no obvious airway distress, great care should be exercised before deciding to delay treatment and perform imaging studies. Management of this condition should occur in pediatric institutions with appropriate medical, surgical, and intensive care facilities.

\section{Disclosure}

The authors report no conflicts of interest in this work.

\section{References}

1. Kirse DJ, Roberson DW. Surgical management of retropharyngeal space infections in children. Laryngoscope. 2001;111(8):1413-1422.

2. Dudas R, Serwint JR. In brief: retropharyngeal abscess. Pediatr Rev. 2006;27(6):e45-e46.

3. Dawes LC, Bova R, Carter P. Retropharyngeal abscess in children. ANZ J Surg. 2002;72(6):417-420.

4. Lalakea MI, Messner AH. Retropharyngeal abscess management in children: current practices. Otolaryngol Head Neck Surg. 1999;121(4):398-405.

5. Al-Sabah B, Bin Salleen H, Hagr A, Choi-Rosen J, Manoukian JJ, Tewfik TL. Retropharyngeal abscess in children: 10-year study. J Otolaryngol. 2004;33(6):352-355.

6. Daya H, Lo S, Papsin BC, et al. Retropharyngeal and parapharyngeal infections in children: the Toronto experience. Int J Pediatr Otorhinolaryngol. 2005;69(1):81-86.

7. Courtney MJ, Mahadevan M, Miteff A. Management of paediatric retropharyngeal infections: non-surgical versus surgical. ANZ J Surg. 2007;77(11):985-987.
International Medical Case Reports Journal

\section{Publish your work in this journal}

The International Medical Case Reports Journal is an international, peer-reviewed open-access journal publishing original case reports from all medical specialties. Previously unpublished medical posters are also accepted relating to any area of clinical or preclinical science. Submissions should not normally exceed 2,000 words or

\section{Dovepress}

4 published pages including figures, diagrams and references. The manuscript management system is completely online and includes a very quick and fair peer-review system, which is all easy to use. Visit $\mathrm{http}: / / \mathrm{www}$.dovepress.com/testimonials.php to read real quotes from published authors.

Submit your manuscript here: https://www.dovepress.com/international-medical-case-reports-journal-journal 